\title{
Malformaciones relacionadas con el síndrome de abdomen en ciruela pasa: serie de casos con revisión de los informes de casos publicados
}

\author{
Bladimir Marín-Montoya ${ }^{1 *}$, Michael Alexander-Vallejo ${ }^{2}$ y Luis A. Robles-Luna1 \\ ${ }^{1}$ Departamento de Pediatría, Servicio de Neonatología; ${ }^{2}$ Departamento de Genética Clínica, Fundación Universitaria de Ciencias de la Salud, \\ Hospital de San José de Bogotá. Bogotá, Colombia
}

\section{Resumen}

Introducción: El síndrome de abdomen en ciruela pasa (SACP) se caracteriza por ausencia de la musculatura de la pared abdominal, alteración del sistema urinario y criptorquidia bilateral. Objetivos: Caracterizar casos de pacientes con diagnóstico de SACP y sus malformaciones relacionadas y buscar en las publicaciones médicas informes en los que se registren casos similares. Metodología: Se describió una serie de casos y se realizó una búsqueda sistemática limitada a informes de casos que cumplieran todos los criterios: defectos de la pared abdominal, alteración del tracto urinario y criptorquidia, y aquellos que consignaran malformaciones adjuntas. Resultados: De 28,452 nacimientos se identificaron 55 casos, ocho con los criterios de inclusión. Todos fueron del género masculino, dos resultaron abortos. Se encontraron malformaciones relacionadas con los sistemas renal, pulmonar, cardiaco y nervioso central. En las publicaciones se tamizaron 1,157 artículos, de los cuales se detectaron 27 casos en 15 informes, con descripción de malformaciones de múltiples sistemas. Conclusión: Es importante evaluar de manera sistemática posibles alteraciones congénitas en otros sistemas, dadas las vinculaciones con otras malformaciones notificadas en este estudio.

Palabras clave: Síndrome de Prune Belly. Síndrome de abdomen en ciruela pasa. Anomalías congénitas. Informes de casos. Recién nacido.

\section{Associated malformations in prune belly syndrome: case series with literature review of case reports}

\section{Abstract}

Introduction: Prune Belly syndrome $(S A C P)$ is characterized by the absence of abdominal wall musculature, alteration of the urinary system and bilateral cryptorchidism. Objectives: Characterize cases of patients diagnosed with PBS and its associated malformations and search the literature for reports where similar cases are mentioned. Methodology: A series of cases were described and a systematic search was carried out limiting the search to case reports that met all the criteria: abdominal wall defects, urinary tract alteration and cryptorchidism, and those that mentioned associated malformations. Results: 55 cases were obtained from 28452 births, 8 met the inclusion criteria. All were male, 2 were abortions. Associated malformations were found at the Renal, Pulmonary, Cardiac and Central Nervous System levels. 1157 articles were screened in the literature, of which 27 cases were detected in 15 reports, with a description of malformations at the level of multiple systems. Conclusion: It is important to systematically evaluate possible congenital alterations in other systems, given the associations with other malformations reported here.

Key words: Prune Belly syndrome. Ciruela Pasa abdomen syndrome. Congenital Abnormalities. Case Reports. Newborn.

\section{Correspondencia:}

*Bladimir Marín-Montoya E-mail: bladi2280@gmail.com
Disponible en internet: 12-11-2021 Perinatol Reprod Hum. 2020;34(1):25-33

www.perinatologia.mx 0187-5337/C 2021. Instituto Nacional de Perinatología Isidro Espinosa de los Reyes. Publicado por Permanyer. Este es un artículo open access bajo la licencia CC BY-NC-ND (http://creativecommons.org/licenses/by-nc-nd/4.0/). 


\section{Introducción}

El síndrome de abdomen en ciruela pasa se caracteriza por la ausencia de la musculatura de la pared abdominal, alteración del sistema urinario y criptorquidia bilateral ${ }^{1,2}$. Se han notificado otras relaciones no genitourinarias en los planos pulmonar, cardíaco, gastrointestinal y musculoesquelético ${ }^{3}$. Es una anomalía muy rara, con una prevalencia global aproximada de 1:35,000-50,000 recién nacidos con mayor presentación en el género masculino en proporción de 18:14,5.

En este país, el Instituto Nacional de Salud solicita notificación obligatoria de todos los casos de malformaciones congénitas; sin embargo, al revisar la base de datos del Departamento Administrativo Nacional de Estadística (DANE), no se hallaron datos acerca de la incidencia y en las publicaciones médicas sólo se registran dos casos colombianos ${ }^{6,7}$.

Este déficit de información suscita preocupación, dada la importancia que tiene su identificación antenatal y su abordaje en el período neonatal, incluido el nacimiento de estos niños en instituciones que cuenten con adecuados recursos humanos y tecnológicos.

La unidad de cuidado intensivo neonatal del Hospital de San José es un centro de referencia para malformaciones congénitas en Bogotá y sus alrededores. Al estudiar los casos de SACP en esta institución puede obtenerse mucha información, que permite identificar otras malformaciones adjuntas, un tema poco estudiado en otras series en el mundo. Esta descripción de los casos se complementó con una revisión sistemática de otros informes de casos en los que se detectó la triada característica y las malformaciones relacionadas ${ }^{8-20}$.

\section{Métodos}

El estudio constó de dos fases. De manera inicial se realizó una búsqueda retrospectiva de todos los casos probables en registros médicos de egresos hospitalarios de la unidad de recién nacidos del Hospital de San José de Bogotá, entre el 1 de enero de 2010 y el 31 de diciembre de 2016. Se detectaron de acuerdo con los códigos CIE-10 relacionados (Q794, Q795, Q792, N329, N369, N338, N289, N134, P839, Q539, Q559, Q606, Q620, Q622, Q638, Q639, Q647, Q793). En estos casos potenciales se confirmó que cumplieran la triada típica (alteración de la musculatura de la pared abdominal, malformación genitourinaria y criptorquidia). Para todos los casos se efectuó una descripción demográfica y las malformaciones adjuntas encontradas. Las variables cualitativas se presentaron mediante proporciones y las cuantitativas con medidas de tendencia central y dispersión.

Para la segunda fase se realizó una búsqueda sistemática con los siguientes descriptores médicos: "Prune Belly Syndrome"[Mesh], "Síndrome de abdomen en ciruela pasa"[DeCs] o "Síndrome de prune belly"[DeCs]. Se utilizaron las siguientes bases de datos: PubMed-LILACS. Se emplearon como criterios de inclusión el tipo de estudio: serie o informe de casos; cumplir la triada completa de SACP; mención de alguna malformación relacionada. Se excluyeron otros tipos de estudios e informes incompletos. La búsqueda la realizó un investigador y los informes se revisaron e incluyeron por criterio de los tres autores. Se valoró la calidad del informe con la lista de revisión para informes de casos publicada JBI Critical Appraisal Tools (Tabla 1). Los datos se incluyeron en una base de datos y se describieron las principales características de cada estudio, incluidas la triada característica del SACP, alteraciones adjuntas, supervivencia y aneuploidías.

\section{Resultados}

Se recogieron 55 casos potenciales y ocho de ellos cumplieron todos los criterios de inclusión (Tabla 2). En las publicaciones médicas se tamizaron 1,157 artículos dentro de los cuales se detectaron 27 casos en 15 informes (Fig. 1).

La frecuencia de presentación del SACP en el Hospital de San José entre los años 2010 y 2016 es de $0.03 \%$, lo que representa 3 pacientes por cada 10,000 embarazos. Todos eran del género masculino, seis fueron gestaciones con recién nacido vivo (75\%) y dos abortos (25\%). En los nacidos vivos, la edad gestacional promedio fue de $36.6 \pm 1.6$ semanas (mínimo 34 y máximo de 39) con una mediana de 37 semanas, con rangos intercuartílicos ubicados en: p25 35.5 semanas, p50 37 semanas, p75 37.5 semanas. Respecto del peso se encontró un promedio de 3,468g (mínimo 3,000 y máximo 3,890 ), con una mediana de 3,490 , con rangos intercuartílicos localizados en p25 3,225 g, p50 3,490 g y p75 3,702 g.

En cuanto a las malformaciones adjuntas (Tabla 3) en los músculos abdominales se identificaron agenesia con una frecuencia de $87.5 \%(n=7)$ y atrofia en un $12.5 \%$ $(n=1)$. Se notificaron alteraciones de las vías urinarias en el 37.5\% ( $n=3)$ como única alteración, en el 50\% $(n=4)$ con dos alteraciones y en el $12.5 \%(n=1)$ con tres tipos de malformaciones. Se encontraron con mayor frecuencia la hidronefrosis en un $42.8 \%$ (grado IV la más frecuente con $28.6 \%$ ), el reflujo vesicoureteral en $21.4 \%$, 


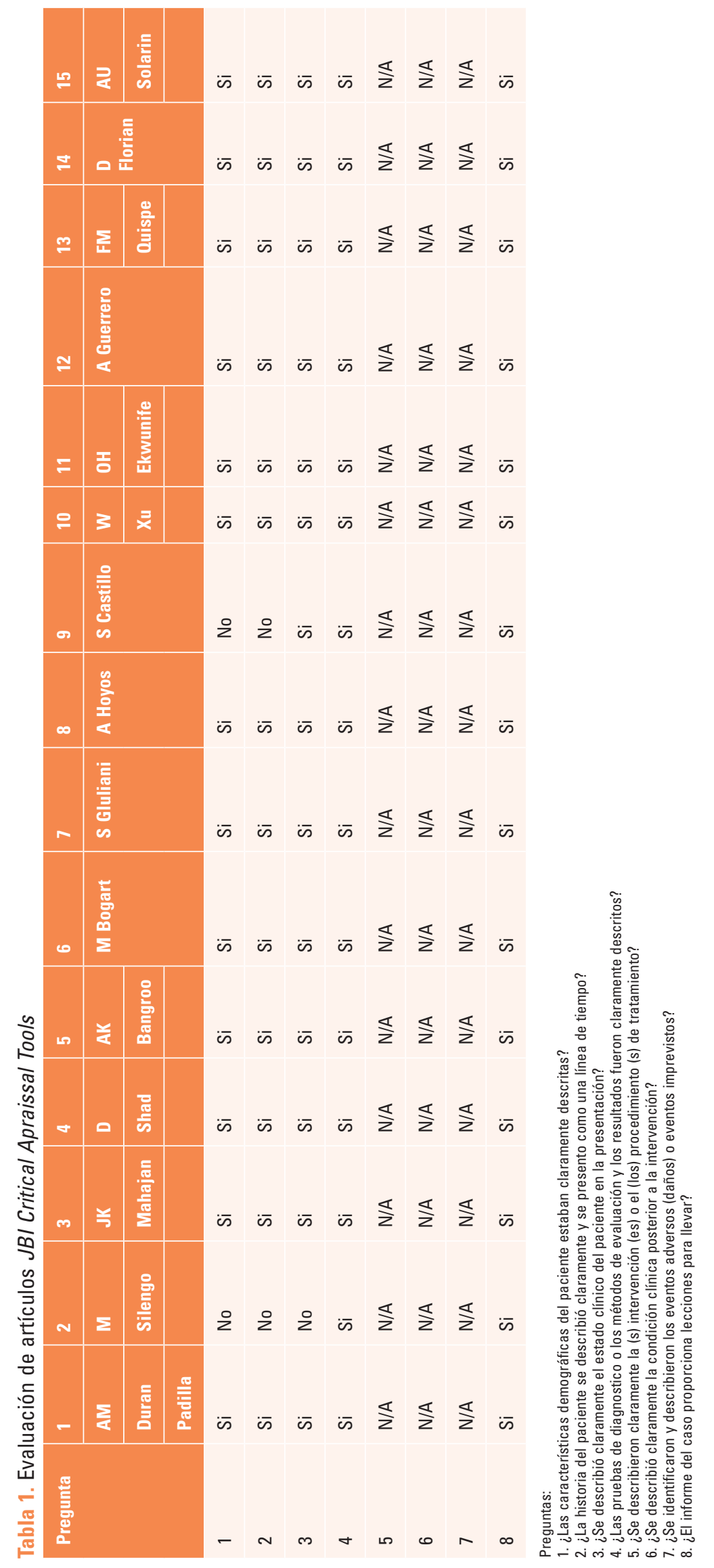




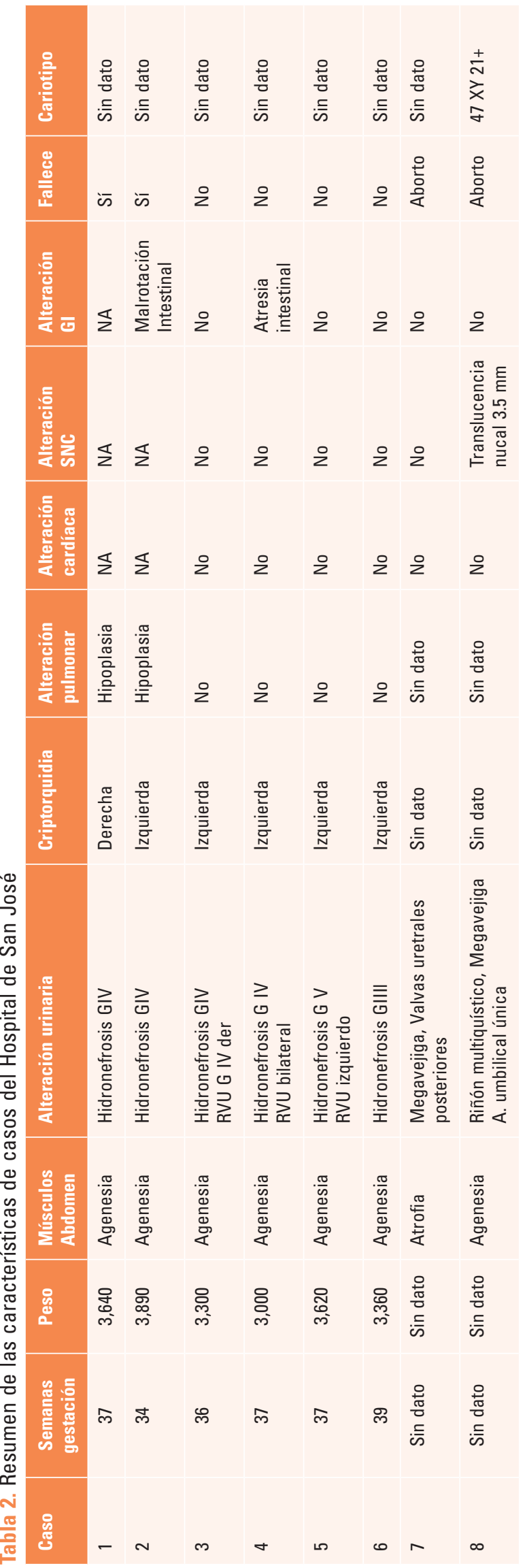

Tabla 3. Frecuencia y porcentaje de alteraciones en casos del Hospital de San José

\begin{tabular}{|c|c|c|}
\hline & Frecuencia & Porcentaje \\
\hline $\begin{array}{l}\text { Músculos abdominales } \\
\text { Agenesia } \\
\text { Atrofia }\end{array}$ & $\begin{array}{l}7 \\
1\end{array}$ & $\begin{array}{l}87.5 \% \\
12.5 \%\end{array}$ \\
\hline $\begin{array}{l}\text { Criptorquidia } \\
\text { Derecha } \\
\text { Izquierda }\end{array}$ & $\begin{array}{l}1 \\
5\end{array}$ & $\begin{array}{l}16.7 \% \\
83.3 \%\end{array}$ \\
\hline $\begin{array}{l}\text { Tipo de malformaciones urinari } \\
\text { Hidronefrosis GIII } \\
\text { Hidronefrosis GIV } \\
\text { Hidronefrosis G V } \\
\text { RVU Izquierdo } \\
\text { RVU Bilateral } \\
\text { Megavejiga } \\
\text { Valvas uretrales posteriores } \\
\text { Riñón multiquístico } \\
\text { A. umbilical única }\end{array}$ & $\begin{array}{l}1 \\
4 \\
1 \\
2 \\
1 \\
2 \\
1 \\
1 \\
1\end{array}$ & $\begin{array}{c}7.1 \% \\
28.6 \% \\
7.1 \% \\
14.3 \% \\
7.1 \% \\
14.3 \% \\
7.1 \% \\
7.1 \% \\
7.1 \%\end{array}$ \\
\hline $\begin{array}{l}\text { Alteración pulmonar } \\
\text { Hipoplasia } \\
\text { Sin Alteración }\end{array}$ & $\begin{array}{l}2 \\
4\end{array}$ & $\begin{array}{l}33.3 \% \\
66.7 \%\end{array}$ \\
\hline $\begin{array}{l}\text { Alteración cardiaca } \\
\text { Sin Alteración }\end{array}$ & 6 & $100 \%$ \\
\hline $\begin{array}{l}\text { Alteración SNC } \\
\text { Translucencia nucal } 3.5 \text { mm } \\
\text { Sin Alteración }\end{array}$ & $\begin{array}{l}1 \\
5\end{array}$ & $\begin{array}{l}16.7 \% \\
83.3 \%\end{array}$ \\
\hline $\begin{array}{l}\text { Alteración gastrointestinal } \\
\text { Malrotación intestinal } \\
\text { Atresia esofágica } \\
\text { Sin alteración }\end{array}$ & $\begin{array}{l}1 \\
1 \\
5\end{array}$ & $\begin{array}{l}14,3 \% \\
14,3 \% \\
71,4 \%\end{array}$ \\
\hline $\begin{array}{l}\text { Fallece } \\
\text { No aplica o aborto } \\
\text { Sí } \\
\text { No }\end{array}$ & $\begin{array}{l}2 \\
2 \\
4\end{array}$ & $\begin{array}{l}25 \% \\
25 \% \\
50 \%\end{array}$ \\
\hline $\begin{array}{l}\text { Cariotipo } \\
\quad 47 \mathrm{XY} 21+\end{array}$ & 1 & $12.5 \%$ \\
\hline
\end{tabular}

megavejiga en $14.3 \%$, valvas uretrales posteriores en $7.1 \%$, riñón multiquístico en $7.1 \%$ y arteria umbilical única en $7.1 \%$. A cuatro pacientes supervivientes se les realizó cistouretrografía miccional y urocultivos que fueron normales, dos de estos casos con lesión renal aguda. Cuatro pacientes fueron objeto de vesicostomía y un paciente se benefició de la nefrostomía. Cuatro casos que egresaron vivos se enviaron a casa con antibioticoterapia profiláctica. En cuanto a malformaciones genitales en los nacidos vivos, la criptorquidia, identificada en todos los casos, fue derecha en un caso e izquierda en los cinco casos restantes.

En el plano pulmonar se registró hipoplasia pulmonar en dos casos. En ninguno de los nacidos vivos se encontraron alteraciones cardíacas ni del sistema 


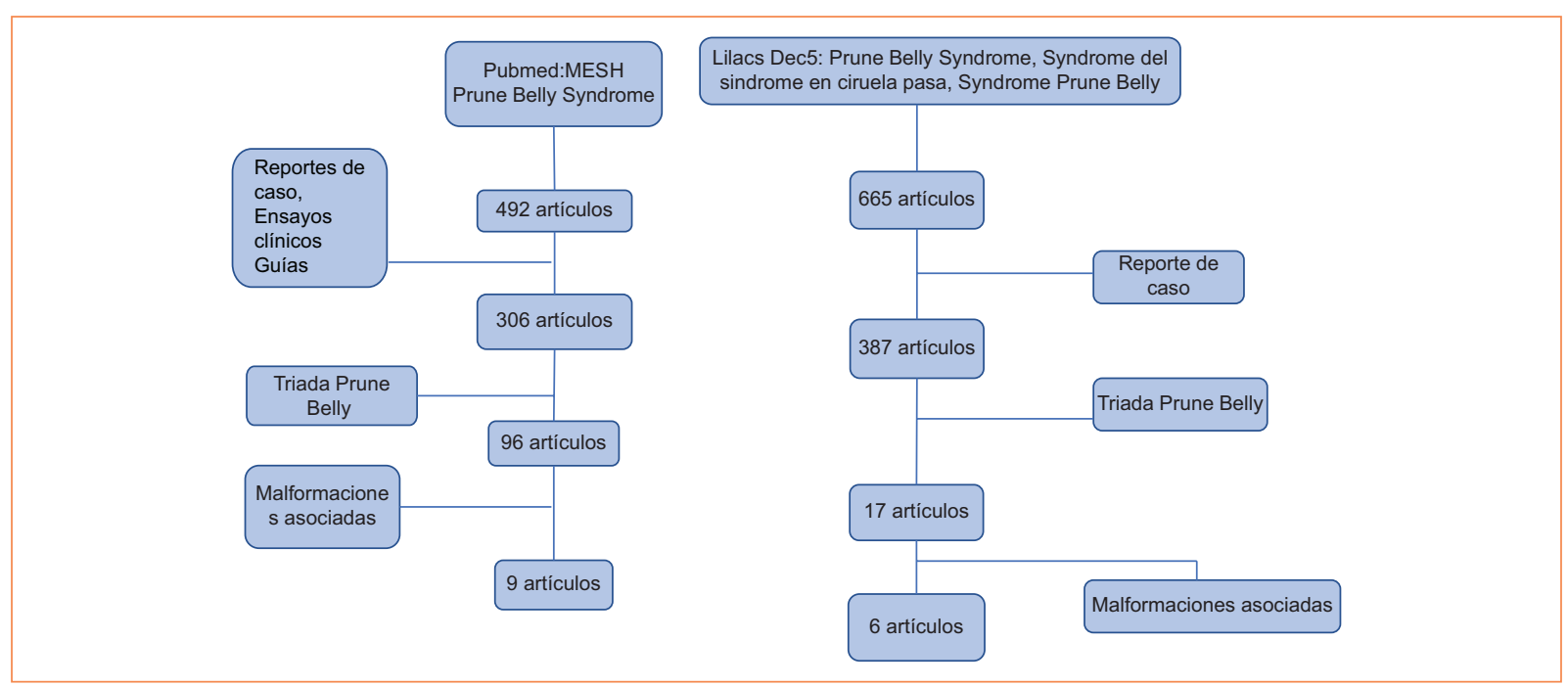

Figura 1. Flujograma de búsquedas sistemáticas.

nervioso central. En el tracto gastrointestinal se reconoció una malrotación intestinal y una atresia esofágica.

La mortalidad en este grupo fue de cuatro casos, dos en el período neonatal y dos abortos. Se realizó cariotipo en todos los casos y se encontró alteración en uno de los casos de aborto con informe de síndrome de Down con una lectura de 47,XY, +21 .

Por otra parte, se identificó a 27 pacientes en las publicaciones médicas, 24 del género masculino $(88.8 \%)$ y 3 del femenino (11.2\%). La totalidad de los casos informados llegó al final de la gestación y se trató en unidades de cuidados intensivos neonatales. La edad gestacional promedio fue de 36.8 semanas \pm 2.8 semanas (mínimo 29 y máximo 40 semanas) con una mediana de 38 semanas y rangos intercuartílicos en p25 36 semanas, p50 38 semanas y p75 39 semanas.

En el sistema renal se encontró que el $100 \%$ tuvo algún tipo de malformación relacionada (Tabla 4), las más frecuentes de las cuales fueron la hidronefrosis $(40 \%)$, la megavejiga y el reflujo vesicoureteral. En uno de los casos del género femenino se registró un nexo con el trastorno de diferenciación sexual. En el plano pulmonar se describió hipoplasia pulmonar en el $26 \%$ de los casos. Desde el punto de vista cardiovascular, en $17.4 \%$ se documentaros anomalías y el conducto arterioso persistente fue el hallazgo más frecuente. En el sistema nervioso central se notificaron anomalías en el $13 \%$, con diversidad de alteraciones estructurales. En el tracto gastrointestinal, el 56.5\% mostró alguna anomalía, en particular malrotación intestinal y atresias. En estos casos se notificó una mortalidad del $20 \%$.

En la Tabla 5 se comparan los resultados obtenidos en los pacientes del Hospital de San José con los informes de casos hallados en las publicaciones médicas.

\section{Discusión}

Al comparar los hallazgos de las publicaciones se puede establecer que las alteraciones pulmonares se presentan en un porcentaje considerable de pacientes: $58 \%$ en las publicaciones médicas y $35 \%$ en los pacientes recopilados de este estudio. La hipoplasia pulmonar es la anomalía de mayor presentación en este siste$\mathrm{ma}^{8,13,14,18}$. Al hablar de alteraciones cardiovasculares, su presentación en las publicaciones es de $25 \%$ y de $14.8 \%$ en el estudio, con el conducto arterioso persistente como el de mayor presentación ${ }^{8,15,16}$. El análisis de las anomalías gastrointestinales permite observar la gran variedad de malformaciones posibles, con una frecuencia de $55 \%$ en este estudio en comparación con el $24 \%$ en las publicaciones de referencia-12,14,17; la malrotación intestinal fue la de mayor presentación en las diferentes series de casos (Tabla 4).

EI SACP se caracteriza por la ausencia de la musculatura de la pared abdominal, alteración del sistema urinario y criptorquidia ${ }^{1}$, y se han notificado casos con otras relaciones no genitourinarias documentadas en diferentes sistemas, entre ellos el pulmonar, cardiaco, gastrointestinal, 
Perinatol Reprod Hum. 2020;34(1)

Tabla 4. Porcentaje de alteraciones relacionadas con el síndrome de abdomen en ciruela pasa

\begin{tabular}{|c|c|c|c|c|c|}
\hline \multicolumn{6}{|c|}{ Resultados de malformaciones en la serie de casos } \\
\hline $\begin{array}{l}\text { Alteraciones } \\
\text { urinarias } \\
77 \%\end{array}$ & $\begin{array}{l}\text { Alteraciones } \\
\text { gastrointestinales } \\
55.2 \%\end{array}$ & $\begin{array}{l}\text { Alteraciones } \\
\text { pulmonares } \\
35.5 \%\end{array}$ & $\begin{array}{l}\text { Alteraciones } \\
\text { cardiacas } \\
14.8 \%\end{array}$ & $\begin{array}{l}\text { Alteraciones del } \\
\text { sistema nervioso } \\
\text { central } \\
11.1 \%\end{array}$ & $\begin{array}{l}\text { Mortalidad en } \\
\text { periodo neonatal } \\
21.2 \%\end{array}$ \\
\hline Hidronefrosis & $\begin{array}{l}\text { Malrotación } \\
\text { intestinal }\end{array}$ & $\begin{array}{l}\text { Hipoplasia pulmonar } \\
\text { bilateral }\end{array}$ & $\begin{array}{l}\text { Conducto } \\
\text { arterioso } \\
\text { persistente }\end{array}$ & Plagiocefalia & Abortos $(n=2.6 .1 \%)$ \\
\hline Megavejiga & Ano imperforado & $\begin{array}{l}\text { Hipoplasia } \\
\text { mediastinal }\end{array}$ & Canal AV & Microcefalia & \\
\hline $\begin{array}{l}\text { Reflujo } \\
\text { vesicoureteral }\end{array}$ & Ano imperforado & Pectus carinatum & $\begin{array}{l}\text { Fístula } \\
\text { arteriocoronaria }\end{array}$ & Hidrocefalia & \\
\hline $\begin{array}{l}\text { Otros (agenesia } \\
\text { tubárica, }\end{array}$ & Atresia esofágica & $\begin{array}{l}\text { Pulmón izquierdo } \\
\text { unilobar y }\end{array}$ & $\begin{array}{l}\text { ventricular } \\
\text { derecha }\end{array}$ & $\begin{array}{l}\text { Agenesia septum } \\
\text { pellucidum }\end{array}$ & \\
\hline ovárica, vaginal) & Colon corto & derecho trilobar & & & \\
\hline Dilatación calicial & Divertículo de Meckel & & & & \\
\hline $\begin{array}{l}\text { Displasia renal } \\
\text { multiquistica }\end{array}$ & Fístula rectovesical & & & & \\
\hline Genitales ambiguos & Hernia inguinal & & & & \\
\hline Megapene & Hernia umbilical & & & & \\
\hline \multicolumn{6}{|l|}{ Megaureteres } \\
\hline \multicolumn{6}{|l|}{ Próstata hipoplásica } \\
\hline \multicolumn{6}{|l|}{$\begin{array}{l}\text { Quiste renal, } \\
\text { hipoplasia renal }\end{array}$} \\
\hline \multicolumn{6}{|l|}{ Uraco persistente } \\
\hline \multicolumn{6}{|l|}{ Uropatia obstructiva } \\
\hline \multicolumn{6}{|l|}{$\begin{array}{l}\text { Válva uretral } \\
\text { izquierda }\end{array}$} \\
\hline \multicolumn{6}{|l|}{$\begin{array}{l}\text { Valva uretral } \\
\text { posterior }\end{array}$} \\
\hline Vejiga dilatada & & & & & \\
\hline
\end{tabular}

\begin{tabular}{|c|c|c|c|c|c|}
\hline \multicolumn{6}{|c|}{ Resultados de malformaciones según la literatura } \\
\hline $\begin{array}{l}\text { Alteraciones } \\
\text { urinarias } \\
53 \%\end{array}$ & $\begin{array}{l}\text { Alteraciones } \\
\text { gastrointestinales } \\
24 \%\end{array}$ & $\begin{array}{l}\text { Alteraciones } \\
\text { pulmonares } \\
58 \%\end{array}$ & $\begin{array}{l}\text { Alteraciones } \\
\text { cardiacas } \\
25 \%\end{array}$ & $\begin{array}{l}\text { Alteraciones del } \\
\text { sistema nervioso } \\
\text { central } \\
5 \%\end{array}$ & $\begin{array}{l}\text { Mortalidad en } \\
\text { periodo neonatal } \\
10-25 \%\end{array}$ \\
\hline Hidronefrosis & $\begin{array}{l}\text { Malrotación } \\
\text { intestinal }\end{array}$ & Hipoplasia pulmonar & $\begin{array}{l}\text { Conducto } \\
\text { arterioso } \\
\text { persistente }\end{array}$ & $\begin{array}{l}\text { Secuelas a largo } \\
\text { plazo }\end{array}$ & Hipoplasia pulmonar \\
\hline $\begin{array}{l}\text { Reflujo } \\
\text { vesicoureteral }\end{array}$ & Atresia intestinal & & $\begin{array}{l}\text { Tetralogia de } \\
\text { Fallot }\end{array}$ & & Prematuridad \\
\hline $\begin{array}{l}\text { Enfermedad renal } \\
\text { quística }\end{array}$ & Torsión esplénica & & $\mathrm{CIA}$ & & \\
\hline $\begin{array}{l}\text { Enfermedad renal } \\
\text { aguda }\end{array}$ & Ano imperforado & & CIV & & \\
\hline Megvejiga & Gastrosquisis & & $\begin{array}{l}\text { Anomalias } \\
\text { valvulares }\end{array}$ & & \\
\hline Hipospadias & Onfalocele & & & & \\
\hline Micropene & Anomalia cloacal & & & & \\
\hline \multicolumn{6}{|l|}{$\begin{array}{l}\text { Anomalías del } \\
\text { uraco }\end{array}$} \\
\hline Ureterocele & & & & & \\
\hline
\end{tabular}


Tabla 5. Comparación de estudios publicados con los resultados obtenidos en el estudio

\begin{tabular}{|c|c|c|c|c|c|c|c|}
\hline Estudios & $\begin{array}{l}\text { No. } \\
\text { casos }\end{array}$ & $\begin{array}{l}\text { Alteración de } \\
\text { pared } \\
\text { abdominal + } \\
\text { defecto renal } \\
\text { + criptorquidia }\end{array}$ & Defecto SNC & $\begin{array}{l}\text { Defecto } \\
\text { cardíaco }\end{array}$ & $\begin{array}{l}\text { Defecto } \\
\text { pulmonar }\end{array}$ & Defecto renal & $\begin{array}{l}\text { Defecto } \\
\text { intestinal }\end{array}$ \\
\hline M Durán Padilla8 & 1 & Sí & $\begin{array}{l}\text { Hidrocefalia } \\
\text { Agenesia } \\
\text { septum } \\
\text { pellucidum }\end{array}$ & $\begin{array}{l}\text { Canal AV } \\
\text { completo }\end{array}$ & $\begin{array}{l}\text { Pulmón } \\
\text { izquierdo } \\
\text { unilobar }\end{array}$ & $\begin{array}{l}\text { Hidronefrosis } \\
\text { Megavejiga }\end{array}$ & $\begin{array}{l}\text { Divertículo } \\
\text { Meckel }\end{array}$ \\
\hline M Silengo ${ }^{9}$ & 1 & Sí & No & No & No & $\begin{array}{l}\text { Hidronefrosis } \\
\text { RVU }\end{array}$ & $\begin{array}{l}\text { Hernia } \\
\text { inguinal } \\
\text { bilateral } \\
\text { Hernia } \\
\text { umbilical } \\
\text { Macroglosia }\end{array}$ \\
\hline $\begin{array}{l}\text { JK Mahajan, } \\
\text { et al. }{ }^{10}\end{array}$ & 1 & Sí & No & No & No & $\begin{array}{l}\text { Hidronefrosis } \\
\text { bilateral } \\
\text { Megavejiga }\end{array}$ & $\begin{array}{l}\text { Ano } \\
\text { imperforado }\end{array}$ \\
\hline D Shah"1 & 1 & Sí & No & No & No & $\begin{array}{l}\text { Agenesia renal } \\
\text { izquierda } \\
\text { Vejiga grande y } \\
\text { engrosada }\end{array}$ & $\begin{array}{l}\text { Atresia } \\
\text { esófago } \\
\text { Ano } \\
\text { imperforado }\end{array}$ \\
\hline AK Bangroo $0^{12}$ & 1 & Sí & No & No & No & $\begin{array}{l}\text { Hidronefrosis } \\
\text { RVU grado V }\end{array}$ & $\begin{array}{l}\text { Ano } \\
\text { imperforado } \\
\text { Colon corto }\end{array}$ \\
\hline MB Bogart et al. ${ }^{13}$ & 2 & Sí & $\begin{array}{l}\text { Espina bífida } \\
\text { oculta }(n=1)\end{array}$ & No & $\begin{array}{l}\text { Hipoplasia } \\
\text { pulmonar } \\
(\mathrm{n}=1)\end{array}$ & $\begin{array}{l}\text { Hidronefrosis } \\
\text { Uropatía } \\
\text { obstructiva }\end{array}$ & No \\
\hline S Giuliani ${ }^{14}$ & 1 & Sí & No & No & $\begin{array}{l}\text { Hipoplasia } \\
\text { pulmonar }\end{array}$ & $\begin{array}{l}\text { Hidronefrosis } \\
\text { bilateral } \\
\text { Megavejiga } \\
\text { Extrofia cloacal }\end{array}$ & Onfalocele \\
\hline A Hoyos ${ }^{6}$ & 1 & Sí & No & No & No & $\begin{array}{l}\text { Megapene } \\
\text { Hidronefrosis } \\
\text { bilateral } \\
\text { Quiste renal } \\
\text { izquierdo } \\
\text { RVU grado III - IV }\end{array}$ & No \\
\hline $\begin{array}{l}\text { S Castillo } \\
\text { Manrique, et al. }{ }^{15}\end{array}$ & 1 & Sí & No & No & No & $\begin{array}{l}\text { Hidronefrosis, } \\
\text { disminución de } \\
\text { parénquima renal } \\
\text { RVU grado V } \\
\text { Uropatía } \\
\text { obstructiva derecha }\end{array}$ & No \\
\hline Wei Xu et al. ${ }^{16}$ & 1 & Sí & No & $\begin{array}{l}\text { Insuficiencia } \\
\text { tricúspide } \\
\text { Fístula AV } \\
\text { coronaria } \\
\text { derecha }\end{array}$ & No & $\begin{array}{l}\text { RVU } \\
\text { Divertículo } \\
\text { vesical }\end{array}$ & No \\
\hline $\begin{array}{l}\text { OH Ekwunife, } \\
\text { et al. }{ }^{17}\end{array}$ & $7 / 9$ & Sí & $\begin{array}{l}\text { Microcefalia } \\
\text { Plagiocefalia }\end{array}$ & No & No & $\begin{array}{l}\text { Hidronefrosis } \\
\text { Megavejiga } \\
\text { RVU }\end{array}$ & $\begin{array}{l}\text { Malrotación } \\
\text { intestinal } \\
\text { Atresia anal } \\
\text { Hernia } \\
\text { umbilical }\end{array}$ \\
\hline A Guerrero ${ }^{7}$ & 1 & Sí & No & No & No & $\begin{array}{l}\text { Hidronefrosis } \\
\text { RVU }\end{array}$ & No \\
\hline FM Quispe et al. ${ }^{18}$ & 2 & Sí & No & No & Hipoplasia & $\begin{array}{l}\text { Hidronefrosis } \\
\text { Megavejiga } \\
\text { RVU }\end{array}$ & No \\
\hline D Florian ${ }^{19}$ & 1 & Sí & No & No & No & Hidrouréter & No \\
\hline AU Solarin 20 & 3 & Sí & No & No & No & RVU & No \\
\hline $\begin{array}{l}\text { Marín B, Vallejo M, } \\
\text { Robles L }\end{array}$ & 8 & Sí & No & No & $\begin{array}{l}2 \text { casos } \\
\text { Hipoplasia }\end{array}$ & $\begin{array}{l}\text { Hidronefrosis } \\
\text { Megavejiga } \\
\text { RVU }\end{array}$ & $\begin{array}{l}\text { Malrotación } \\
\text { intestinal } \\
\text { Atresia } \\
\text { esofágica }\end{array}$ \\
\hline
\end{tabular}




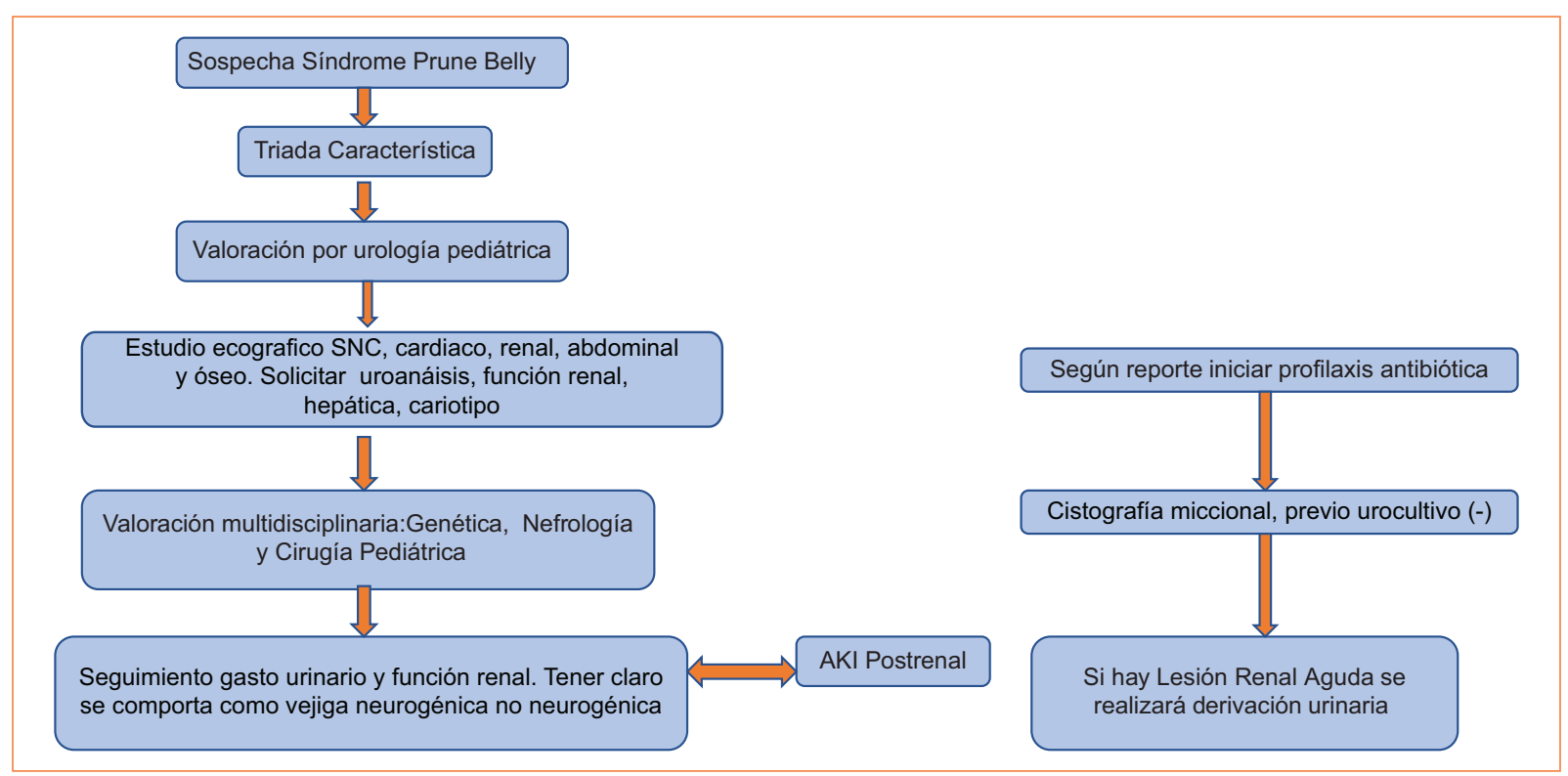

Figura 2. Flujograma diagnóstico y seguimiento renal.

musculoesquelético ${ }^{2}$. Se calcula una prevalencia global de 1:35,000-50,000 recién nacidos, con mayor presentación en el género masculino en proporción de 18:1.

La causa del SACP aún se desconoce; dos teorías han propuesto el desarrollo de este síndrome: la obstrucción uretral y el defecto en el desarrollo mesodérmico. La primera causa distensión vesical y dilatación del tracto urinario del feto en desarrollo y es la distensión la que evita el desarrollo normal de la musculatura abdominal y el descenso testicular ${ }^{3}$. Esta teoría se relaciona perfectamente con los casos de SACP, cuya característica es la atresia uretral, pero no explica la presencia de valvas uretrales posteriores. Se ha postula como posible mecanismo de aparición de las valvas uretrales posteriores la canalización tardía de la uretra durante las semanas 11 y 16 de gestación, así como la obstrucción uretral transitoria en algún momento del desarrollo. Una hipótesis es la presencia de próstata hipoplásica que aparece debido a un estímulo aberrante focal en el desarrollo urogenital del seno mesodérmico; esta alteración puede causar una membrana uretral que puede comportarse con un mecanismo de valva que propicia una obstrucción uretral transitoria.

Las complicaciones urinarias relacionadas con el SACP se originan durante el desarrollo embrionario y fetal. Éstas incluyen agenesia renal, riñón en herradura, uréter ectópico, enfermedad quística renal y duplicación del tracto urinario ${ }^{4}$.

Se encontró que el SACP posee un alto grado de expresividad atribuible a la variabilidad en los fenotipos distintos de la triada habitual descrita: alteración de la musculatura abdominal, genitourinaria y criptorquidia. Estos defectos adjuntos se encuentran en los planos cardiaco, pulmonar, gastrointestinal, nervioso central y osteomuscular. También se han descrito diversas mutaciones en grupos familiares, lo que podría constituir alta heterogeneidad genética. Aunque no es claro el patrón de herencia mendeliana, es la autosómica recesiva la de mayor descripción en las publicaciones médicas. La mutación homocigota en el gen del receptor colinérgico muscarínico MCHR3, localizado en el brazo largo del cromosoma 1 banda 43 (1q43), se ha descrito como la alteración genómica que explica la alteración en los órganos que intervienen en el SACP. Adicionalmente, los trastornos relacionados con el gen ACTG2 demuestran un subconjunto de miopatía visceral que afecta a la vejiga y al intestino. Por otra parte, la deficiencia de $\alpha 1$-antitripsina puede ser un factor etiológico en la aparición de SACP en relación con la alteración pulmonar. Asimismo, se ha referido la relación entre el SACP y el síndrome de Beckwith-Wiedemann debido a la anomalía del tracto urinario por la desmetilación aislada del gen KCNQ10T1. Murray demostró una deleción del factor-1 $\beta$ de los hepatocitos nucleares en un paciente con SACP; esta mutación sugiere la aparición y relación de alteraciones renales y metabólicas. Debe señalarse que no hay relación genética directa entre el SACP y la aparición de cardiopatía y alteración en el sistema nervioso central ${ }^{1-3,5}$.

Ante la pobre evidencia que explica la etiología del SACP, desde el punto de vista genético es posible 
empezar a abrir camino hacia la investigación de un posible pleiotropismo relacionado y por lo cual se deben evaluar de manera sistemática posibles alteraciones en los planos neurológico, cardiaco, pulmonar, gastrointestinal y óseo, entre otros. De modo adicional, se logró observar que no todos los pacientes cursan con las mismas complicaciones adjuntas, quizá en relación con la genética de poblaciones, lo que lleva a determinar las características en ciertas regiones.

Por último, debe sensibilizarse al cuerpo médico, en especial quienes establecen el diagnostico antenatal, seguimiento y abordaje posnatal, con el fin de incrementar la sospecha e identificar posibles malformaciones adjuntas de este síndrome. Se sugiere un flujograma diagnóstico que incluya hallazgos distintos de la triada típica y seguimiento para esquematizar la evaluación por sistemas en esta enfermedad (Fig. 2).

\section{Conclusiones}

El síndrome de abdomen en ciruela pasa, a pesar de ser una entidad rara, y de acuerdo con la casuística hallada del Hospital de San José, es un referente en el diagnóstico y tratamiento. Se deben buscar alteraciones en otros sistemas, ya que el posible pleiotropismo genético puede ocasionar malformaciones distintas de la triada característica.

\section{Agradecimientos}

Los autores agradecen a los servicios de Pediatría, Genética clínica, Archivo y Estadística del Hospital de San José.

\section{Financiamiento}

La presente investigación no ha recibido ayuda específica proveniente de agencias del sector público, sector comercial o entidades sin ánimo de lucro

\section{Conflicto de intereses}

Los autores declaran no tener conflicto de intereses que pueda afectar la imparcialidad de esta investigación.

\section{Responsabilidades éticas}

Protección de personas y animales. Los autores declaran que los procedimientos seguidos se conformaron a las normas éticas del comité de experimentación humana responsable y de acuerdo con la Asociación Médica Mundial y la Declaración de Helsinki.

Confidencialidad de los datos. Los autores declaran que han seguido los protocolos de su centro de trabajo sobre la publicación de datos de pacientes.

Derecho a la privacidad y consentimiento informado. Los autores declaran que en este artículo no aparecen datos de pacientes.

\section{Bibliografía}

1. Seidel NE, Arlen AM, Smith EA, Kirsch AJ. Clinical manifestations and management of prune-belly syndrome in a large contemporary pediatric population. Urology. 2015;85:211-215

2. Smolkin T, Soudack M, Goldstein I. Prune belly syndrome: expanding the phenotype. Clin Dysmorphol. 2008;17(2):133-5.

3. Meimer I, Bar-Ziv J, Katz M. Prenatal ultrasonic diagnosis of the extreme form of prune belly syndrome. J Clin Ultrasound. 1985;13(8):581-3.

4. Simons SHP, van Dijk M, Anand KS, Roofthooft D. Do we still hurt newborn babies? Arch Pediatr Adolesc Med. 2003;157(11):1058.

5. Taghavi K, Sharpe C, Stringer MD. Fetal megacystis: a systematic review. J Pediatr Urol. 2017;13(1):7-15.

6. Hoyos A, Botero P. Síndrome prune belly (vientre en ciruela): reporte de un caso. Medicina UPB. 2010;29(2):155-161.

7. Guerrero AF, Cuadros CA, Archila DC. Síndrome de prune belly: presentación de un caso y revisión de la literatura. Rev Salud Uis. 2010;42(1).

8. Durán MA, Solís V. Síndromes VACTER-H y prune belly. Rev Med Hos Gen Méx. 2000;63(3):196-9.

9. Watanabe H, Yamanaka T. A possible relationship between Beckwith-Wiedemann syndrome, urinary tract anomaly and prune belly syndrome. Clin Genet. 1990;38(6):410-4.

10. Mahajan JK, Ojha S, Rao W. Prune-belly syndrome with anorectal malformation. Eur J Pediatr Surg. 2004;14(5):351-4

11. Shah D, Sharma S, Mishra K, Report C. Case reports VACTERL Association with Prune-Belly Syndrome. Indian Pediatr. 2004;2-4.

12. Bangroo AK, Tiwari S, Khetri R. Congenital pouch colon with prune belly syndrome and megalourethra. Pediatr Surg Int. 2005;21(6):474-7.

13. Bogart MM, Arnold HE, Greer KE. Prune-belly syndrome in two children and review of the literature. Pediatr Dermatol. 2006;23(4):342-5.

14. Giuliani S, Vendryes C, Malhotra A. Prune belly syndrome associated with cloacal anomaly, patent urachal remnant, and omphalocele in a female infant. J Pediatr Surg. 2010;45(11):e39-42.

15. Spagnoli N, Castillo S. Síndrome de prune belly: reporte de un caso. Rev Medicina. 2010;16(1):59-63.

16. Xu W, Wu H, Wang DX, Mu ZH. A case of prune belly syndrome. Pediatr Neonatol. 2015;56(3):193-6.

17. Ekwunife $\mathrm{OH}$, Ugwu JO, Modekwe V. Prune belly syndrome: early management outcome of nine consecutive cases. Nigerian $\mathrm{J}$ Clin Pract. 2014;17(4):425-30.

18. Maita F, Panozo S, Verástegui D, Hochstatter E. Síndrome de prune belly: diagnóstico y manejo pre y posnatal. Presentación de dos casos. Gac Médica Boliv. 2013;36(1):35-8.

19. Villegas J, Moreno M, Florian D. Síndrome de prune belly : diagnóstico y manejo. Pediatr Panama. 2015;44(2):28-35.

20. Solarin AU, Disu EA, Gbelee HO. Three cases of prune belly syndrome at the Lagos State University Teaching Hospital, Ikeja. Saudi J Kidney Dis Transpl. 2018;29(1):178-84. 